\title{
Molecular crowding effects on the distribution of amphiphiles in bio- logical media
}

Patrícia A. T. Martins', Neuza Domingues, ${ }^{1,2}$, Cristiana Pires ${ }^{1}$, Ana Maria Alves', Tiago Palmeira ${ }^{1,3}$, Jaime Samelo ${ }^{1}$, Renato Cardoso $^{1,4}$, Adrian Velazquez-Campoy, ${ }^{5,6}$ and Maria João Moreno ${ }^{1^{*}}$

1 CQC-Biological Chemistry Group, Chemistry Department FCTUC, Largo D. Dinis, Rua Larga, 3004-535 Coimbra, Portugal

2 CEDOC, NOVA Medical School | Faculdade de Ciências Médicas, Universidade NOVA de Lisboa, 1169-056 Lisboa, Portugal

3 CQFM-IN and IBB-Institute for Bioengineering and Biosciences, Instituto Superior Técnico, Universidade de Lisboa, Lisboa, Portugal

4 Exogenus Therapeutics S.A, Biocant Park, 306o-197 Cantanhede, Portugal

5 Institute of Biocomputation and Physics of Complex Systems (BIFI), Joint Units IQFR-CSIC-BIFI and GBsCCSIC-BIFI, and Department of Biochemistry and Molecular and Cell Biology, Universidad de Zaragoza, 50018 Zaragoza, Spain

6 Aragon Institute for Health Research (IIS Aragon), 50009 Zaragoza, Spain; Biomedical Research Networking Centre for Liver and Digestive Diseases (CIBERehd), Madrid, Spain; Fundacion ARAID, Government of Aragon, 50018 Zaragoza, Spain

KEYWORDS. Molecular crowding effects; Amphiphiles; Solubility; Equilibrium distribution; Kinetics; Pharmacokinetics.

This manuscript has 6498 words, and 8 Figures.

\footnotetext{
ABSTRACT: Biological systems are the result of the interactions established among their many distinct molecules and molecular assemblies. The high concentration of small molecules dissolved in the aqueous media alter the water properties with important consequences in the interactions established. In this work, the effects of high concentrations of the disaccharide trehalose on the solubility of a homologous series of fluorescent amphiphiles (NBD-Cn, n=4-16) and on their interaction with a lipid bilayer and a serum protein is quantitatively characterized. Both kinetic and equilibrium aspects are reported for a better understanding of the effects observed.

The aqueous solubility of the most hydrophobic amphiphiles $(n \geq 8)$ is strongly increased by 1 M trehalose, while no significant effect is observed for the most polar amphiphile $(n=4)$. This results from a decrease in the magnitude of the hydrophobic effect at conditions of molecular crowding. A small decrease is observed on the equilibrium association with serum albumin. This is most significant for amphiphiles with longer alkyl chains, in agreement with their increased solubility in the aqueous media containing trehalose.

The effects on the association of the amphiphiles with lipid bilayers are influenced by both equilibrium and kinetic aspects. On one hand, the decreased magnitude of the hydrophobic effect leads to a decrease in the affinity of the amphiphiles towards the membrane. However, this tendency may be overbalanced by the effects on the kinetics of the interaction (insertion/desorption) due to the increase in the viscosity of the aqueous media.

It is shown that the distribution of amphiphilic drugs in the crowded biological media is significantly different from that predicted from studies in dilute solutions and that the effects are dependent on solute's hydrophobicity.
} 
Biological fluids are very crowded systems, with a high concentration of small molecules, macromolecules and supramolecular assemblies. In these conditions, a significant fraction of the solvent is solvating the solutes instead of free in solution and therefore, its properties are strongly altered. Media properties such as solubility and interaction with binding agents are of very high relevance for the availability and effect of biological ligands and pharmacological agents. These properties are usually evaluated through the utilization of model systems that should be as simple as possible, while presenting the relevant characteristics of the in vivo system. Molecular crowding is an important characteristic of the in vivo system whose effects are at present not fully understood, at least in quantitative terms.

The effects of molecular crowding on the structural properties of proteins have been thoroughly characterized, namely by Timasheff and co-workers. ${ }^{1-7}$ High concentrations of neutral solvent additives have been shown to stabilize the compact native structure of globular proteins against thermal denaturation. ${ }^{4}$ This effect is due to an exclusion of the solvent additives (co-solvents) from the protein-solvent interface, which stabilizes the state with the smaller interface - the compact native state. This is likely one of the important effects of the high concentration of non-reducing sugars generated by living systems in stress conditions.

In contrast with the strong effects observed on the thermal stability of proteins, very small effects are observed on the properties of membranes in conditions of excess water. Only a small increase in the melting temperature of phosphatidyl choline multilamelar vesicles has been observed with negligible effects on unilamelar vesicles. ${ }^{8}$ Similar results have been obtained by others. ${ }^{9,10}$ A small decrease in the dipole potential have also been observed in monolayers in the presence of trehalose." In addition, Molecular Dynamics simulations have shown that at high concentrations, trehalose interacts with the polar head-groups of the lipids replacing some of the water molecules, but with little effects on the properties of the lipid bilayer (area per lipid, acyl chain order or dipole potential). ${ }^{12-15}$ Non-reducing sugars are however known to be of fundamental importance to protect cell membranes under stress conditions generated by dehydration. This is mostly due to the solvation of the lipid interface by the co-solvent, which prevents the lipid bilayer from attaining dehydrated crystalline phases. ${ }^{16,17}$

The different effects of molecular crowding generated by disaccharides on proteins and lipid membranes in conditions of excess water, may be due to the properties of the interfaces with the solvent. Proteins are small and have rough surfaces, in contrast with the smooth surface of lipid bilayers. The polarity and charge distribution at their surfaces are also very different, with the lipid bilayers being homogeneous while proteins present patches of distinct polarity and charge. Those differences lead to distinct interactions with the solvent, and they are therefore affected uniquely by the altered properties of the solvent. ${ }^{18}$

Another important aspect of the presence of high concentrations of disaccharides in biological fluids is their effect on the solubility of biological ligands and pharmacological agents. In this respect, the polarity of the molecule of interest is of particular relevance, namely its amphiphilic properties. Polar groups which interact strongly with water (notably charged groups) are expected to interact less efficiently with aqueous solutions containing high concentrations of disaccharides. On the other end, non-polar groups may become more soluble due to the effects of the disaccharides on water properties. The effect of high concentrations of trehalose on the solubility of alcohols of different alkyl chain length supports the above interpretation, with a significant decrease in the heat capacity compatible with a decrease in the hydrophobic solvation. ${ }^{19}$

When compared with other disaccharides, trehalose presents several characteristics that justify its preferential use by several micro-organisms as a stress protection agent. ${ }^{20}$ Among them it should be highlighted trehalose inertness and the prevalence of open conformations leading to few intramolecular interactions and preferential interaction with other molecules. ${ }^{21,22}$ Trehalose is therefore the disaccharide used in this study to mimic the molecular crowding effects generated by high concentrations of small molecules in biological fluids.

In spite of the large amount of work done on the effects of disaccharides on the properties of water and on the structure and stability of macromolecules and supramolecular assemblies, little is known regarding the effects on the interaction of ligands with binding agents in physiological conditions. The purpose of this work is to contribute to this goal, with the quantitative characterization of the aqueous solubility, association with a lipid bilayer and with albumin. A homologous series of amphiphiles with increasing alkyl chain length is studied, to gain insight regarding the contribution from ligand polar and non-polar groups. Both the kinetics and the equilibrium of the interactions are reported for a better understanding of the effects observed. The results obtained in this work are of high relevance in the prediction of the local availability of drugs and biological ligands at their target sites, and therefore on their activity and effectiveness.

\section{Materials and Methods}

Materials. 1-Palmitoyl-2-oleoyl-sn-glycero-3-phosphocholine (POPC) was from Avanti Polar Lipids, Inc. (Alabaster, Alabama, USA), and bovine serum albumin (BSA) was from Applichem (Darmstadt, Germany). The fluorescent amphiphiles, NBD-Cn, were synthesized and purified as described previously. ${ }^{23}$ Trehalose was from starch, Sigma ( $\geq 99 \%$ pure), and all other reagents and solvents were of analytical grade, or higher purity, from Sigma-Aldrich Química S.A. (Sintra, Portugal). The pH was controlled by the buffer HEPES, and was prepared at a total concentration of $10 \mathrm{mM}, \mathrm{pH}=7.4$, with $0.02 \% \mathrm{NaN}_{3}, 1 \mathrm{mM}$ EDTA, and $150 \mathrm{mM} \mathrm{NaCl}$ (hereafter designed simply by buffer). The aqueous solutions containing trehalose at a concentration of $1 \mathrm{M}$ (buffer + trehalose) were prepared via the dissolution of the necessary amount of all solid components in the amount of water needed to perform the total required volume. The solutions were filtered through a $1 \mu \mathrm{m}$ nylon filter and stored at $4{ }^{\circ} \mathrm{C}$.

Spectroscopic Equipment. Steady state fluorescence measurements were performed on a Cary Eclipse fluores- 
cence spectrophotometer (Varian) equipped with a thermostatted multicell holder accessory and automatic polarizers. UV-vis absorption was performed on a Unicam UV530 spectrophotometer (Cambridge, U.K.). Stoppedflow measurements were performed on a thermostatted stopped-flow fluorimeter (Hi-Tech model SF-61). Data was analyzed using Microsoft Excel and Solver.

Solubility in Aqueous Solutions. The solubility of the monomeric form of NBD- $C_{n}$ in buffer (with and without trehalose) was assessed at $25{ }^{\circ} \mathrm{C}$ via deviations to linearity in the amphiphile absorption and/or fluorescence as a function of the total concentration of amphiphile, as described previously ${ }^{23}$.

Binding to Bovine Serum Albumin. The equilibrium association constant of the amphiphiles to the higher affinity binding site on BSA was obtained through the addition of aliquots from a $600 \mu \mathrm{M}$ solution of BSA in buffer, into a solution of the amphiphile. The amphiphile was first dissolved in methanol at the necessary concentration and a small aliquot was squirted into the aqueous buffer (with or without trehalose), while stirring gently with a vortex apparatus, to obtain the required final concentration of NBD-Cn and $0.5 \%$ methanol. Aliquots of a BSA solution at $600 \mu \mathrm{M}$ were added to the amphiphile solution and it was allowed to equilibrate for 5 minutes at the desired temperature before reading the fluorescence spectra. Fluorescence intensity was corrected for dilution due to the addition of BSA, and the absorption at the excitation wavelength was always smaller than o.1, to guarantee linearity between the fluorescence intensity and fluorophore concentration. The concentration of BSA was verified by absorption, considering a molar absorption coefficient $\varepsilon=4.4 \times 10^{4} \mathrm{M}^{-1} \mathrm{~cm}^{-1}$ at $279 \mathrm{~nm}^{24}$

Partition Coefficients between the Aqueous Phase and POPC Bilayers using fluorescence. The aqueous solution containing the amphiphile was prepared as above, although to a final concentration of methanol equal to $1 \%$. Those were then mixed with an equal volume of POPC solution in the aqueous buffer (without methanol), at different lipid concentrations and allowed to equilibrate for 1 to $2 \mathrm{~h}$ at $25^{\circ} \mathrm{C}$. The POPC was in the form of large unilamelar vesicles (LUV) that were prepared by evaporation of a POPC solution in the azeotropic mixture of chloroform and methanol $(87: 13, \mathrm{v}: \mathrm{v})$ followed by hydration of the film with the aqueous buffer and subsequent extrusion (Extrusor from Lipex Biomembranes, Vancouver, British Columbia, Canada) through $100 \mathrm{~nm}$ pore size filters (Nucleopore, Whatman, Springfield Hill, U.K.), as described previously $^{25}$. The final concentration of POPC was verified using a modified version of the Bartlett phosphate assay. The samples containing trehalose required previous treatment due to reaction of perchloric acid with trehalose. The LUVs in aqueous solution were added to an RP18 column $(2.5 \mathrm{~cm}$ high, $1.5 \mathrm{~cm}$ diameter) and the salts and trehalose were eluted using $8 \mathrm{ml}$ water. The lipid was then eluted with 6 $\mathrm{mL}$ methanol, the solvent was evaporated and the lipid film was resuspended in water to proceed with the usual phosphate quantification assay.

The total concentration of the fluorescent amphiphiles was equal to, or smaller than, half the value of its critical aggregation concentration (CAC) in the aqueous solution, and the ratio of POPC to bound amphiphile was higher than 50 to guaranty that the properties of the bilayer are not affected by the ligand. ${ }^{26-28}$

Partition Coefficients between the Aqueous Phase and POPC Bilayers using ITC. Titrations were performed on a VP-ITC instrument from MicroCal (Northampton, MA, USA) with an injection speed of $0.5 \mu \mathrm{l} \mathrm{s}^{-1}$, stirring speed $459 \mathrm{rpm}$, and reference power $10 \mu \mathrm{cal} \mathrm{s}^{-1}$. As recommended by the manufacturer, a first injection of $4 \mu \mathrm{l}$ was performed, followed by additions of $10 \mu$ l per titration step. To overcome possible solute loss due to binding to equipment parts during sample manipulation, the sample cell was pre-rinsed with a solution with the same composition as the solution required in the titration. ${ }^{27,28}$ All solutions were previously degassed for $15 \mathrm{~min}$.

Rate of interaction of NBD-Cn with POPC Bilayers, and translocation between leaflets. The rate of insertion/desorption was characterized for NBD- $\mathrm{C}_{10}$ and NBD$\mathrm{C}_{14}$, in the temperature range from 15 to $30{ }^{\circ} \mathrm{C}$. The moderate solubility of NBD- $\mathrm{C}_{10}$ in the aqueous media allows the direct characterization of its interaction with the POPC LUVs. A fresh solution of the amphiphile in buffer was prepared by squirting a small aliquot of a solution of amphiphile in methanol while stirring gently with a vortex apparatus (final methanol concentration equal to $0.5 \%$ ). This solution was then mixed (using the stopped-flow equipment) with an equal volume of solutions of POPC in the same buffer, and the increase in NBD fluorescence due to binding to the POPC bilayer followed. The rate of transfer to the LUVs was characterized at several concentrations of lipid, and the rate constant of insertion and desorption was obtained from the global best fit. The low aqueous solubility of NBD- $\mathrm{C}_{14}$ does not allow the direct transfer from the aqueous media into the LUVs. In this case, the fluorescent amphiphile was first equilibrated with BSA (following the procedure described above), and this solution was mixed with POPC LUVs in the stopped flow apparatus. The characteristic rate constant for transfer from BSA to the LUVs was obtained at different lipid concentrations, and the rate constants of insertion/desorption were obtained from the slope/intercept respectively. ${ }^{29}$

The kinetics of translocation of the amphiphiles was followed via the irreversible quenching of NBD by dithionite added to the aqueous solution outside the lipid vesicles, as described previously. ${ }^{29} \mathrm{NBD}-\mathrm{Cn}$ was added to the lipid solution before the preparation of the lipid film and allowed to equilibrate for at least $15 \mathrm{~min}$ with occasional vortex. The molar ratio of NBD-Cn to POPC was always smaller than $0.1 \%$.

\section{Results and discussion}

Solubility of NBD- $\mathrm{C}_{\mathbf{n}}$ in the aqueous media. The critical aggregation concentration (CAC) of NBD- $C_{n}$ in the aqueous buffer without trehalose has been previously measured, being $2.5 \times 10^{-4} \mathrm{M}$ for NBD- $\mathrm{C}_{4}$ and $5.0 \times 10^{-8} \mathrm{M}$ for for NBD-C 10 , at $25^{\circ} \mathrm{C}^{23}$ At the CAC, the absorption of NBD$\mathrm{C}_{4}$ at the maximum of the lower energy band is equal to 5.2, for $\varepsilon=2.1 \times 10^{4} \mathrm{M}^{-1} \mathrm{~cm}^{-1}$ and $\mathrm{l}=\mathbf{1} \mathrm{cm}$. At those high absorptions, inner filter and geometrical effects do not allow the 
use of fluorescence to quantitatively evaluate the amount of soluble amphiphile. The CAC was therefore characterized through the dependence of absorption with amphiphile concentration (using the cell pathlength required to guaranty linearity between absorption and concentration), and this was also the method used for NBD- $\mathrm{C}_{6}$. In contrast, at the CAC of NBD- $\mathrm{C}_{8}$ in buffer without trehalose $\left(9.1 \times 10^{-7} \mathrm{M}\right)^{23}$ the absorption is equal to 0.02 . The dependence of the fluorescence intensity on the amphiphile concentration was the method used to quantitatively characterize their CAC. The results obtained are shown in Figure 1 , where they are compared with the CAC previously obtained in the absence of trehalose.

The absorption spectra and molar extinction coefficient of NBD- $C_{n}$ was not affected by the presence of trehalose. In contrast, a small but significant increase in the fluorescence quantum yield was observed (around 20\%, results not shown). The effect of $0.5 \mathrm{v} / \mathrm{v} \%$ methanol was also evaluated for NBD- $\mathrm{C}_{4}$, leading to negligible variations, both in its absorption and fluorescence. This is in agreement with the considerably higher size and concentration of trehalose (1 $\mathrm{M}$ as compared with $0.12 \mathrm{M}$ that corresponds to $0.5 \mathrm{v} / \mathrm{v}$ $\%$ methanol).

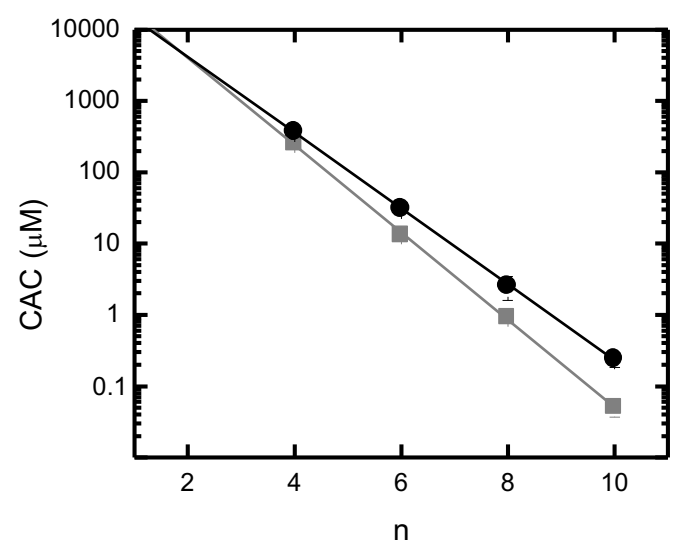

Figure 1 - Dependence of NBD- $\mathrm{C}_{\mathrm{n}}$ critical aggregation concentration (CAC) on the length of the alkyl chain (n), in HEPES buffer containing $1 \mathrm{M}$ trehalose (,). The average of 3 to 6 independent experiments is shown, the standard deviation is also represented, being in most cases smaller than the symbol size. The results are compared with data from literature in the absence of trehalose $(!)^{23}$.

The presence of trehalose increases the solubility of all amphiphiles studied ( $\mathrm{n}=4$ to 10$)$, the effect being significantly larger for longer alkyl chains (CAC(trehalose)/ $\mathrm{CAC}(\mathrm{HEPES})=2,3,4$ and 6). From the trend obtained (Figure 1 ), it is predicted that for $\mathrm{n}=\mathbf{2}$ the solubility is the same in both solvents $(5 \mathrm{mM})$, and for smaller alkyl chains a lower solubility in the buffer containing trehalose is expected. A similar trend was observed before for an homologous series of primary alcohols $(n=4$ and 5$) \cdot{ }^{19}$ However, the solubility of the alcohols was smaller in the trehalose containing solution $(\mathrm{CAC}$ (trehalose $) / \mathrm{CAC}(\mathrm{HEPES})=0.2$ and 0.4 , for $n=4$ and 5 , respectively), with $n=6$ being the predicted alkyl length at which the solubility in both solvents would be the same ( $35 \mathrm{mM})$. For both homologous series it is observed a null effect of trehalose in the aqueous solubility of solutes with intermediate polarity. The presence of trehalose decreases the solubility of very polar solutes, and increases that of non-polar solutes.

Association of NBD- $\mathrm{C}_{\mathbf{n}}$ with serum albumin. The association of NBD- $C_{n}(n=4,6$, and 8) with bovine serum albumin (BSA) was followed via the increase in the fluorescence intensity of NBD- $\mathrm{C}_{\mathrm{n}}{ }^{23}$ The presence of trehalose does not affect significantly the fluorescence spectra and quantum yield of NBD- $\mathrm{C}_{4}$ when associated with BSA. However, an increase of $40 \%$ was observed in the fluorescence quantum yield of NBD- $\mathrm{C}_{8}$ when the aqueous media contained $1 \mathrm{M}$ trehalose, and an intermediate behavior was obtained for NBD- $\mathrm{C}_{6}$ (results not shown). The sensitivity to the composition of the aqueous media when bound to BSA indicates that the NBD group is exposed to the aqueous media, although it may also reflect changes in the structure of BSA in the presence of trehalose. ${ }^{4,7}$

The dependence of the NBD fluorescence intensity with the concentration of BSA is shown in Figure 2. The results were well described by a model considering association with a single binding site, from which the equilibrium association constant $\left(K_{\mathrm{B}}\right)$ to the binding site with highest affinity was obtained. ${ }^{23}$ The results show that the presence of trehalose does not alter the value of $K_{\mathrm{B}}$ obtained for NBD$\mathrm{C}_{4}$, and a very small (non-significant) decrease is observed for NBD-C 6 . The affinity of NBD-C 8 to BSA is however decreased by $40 \%$ when $1 \mathrm{M}$ trehalose is added to the aqueous media (from $5.8 \times 10^{3}$ to $3.5 \times 10^{3}$ ). This result was expected, given the higher solubility of $\mathrm{NBD}-\mathrm{C}_{8}$ in the aqueous media containing trehalose. The decrease in $K_{\mathrm{B}}$ is however smaller than the increase observed in the aqueous solubility, suggesting that the interactions between the amphiphile and BSA were also influenced by the presence of trehalose, possibly through changes in the protein structure.

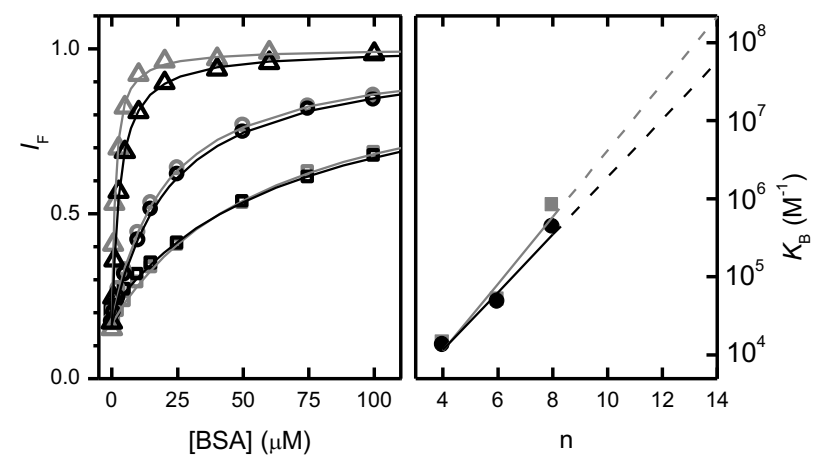

Figure 2 - Fluorescence intensity of NBD-C $C_{4}(\forall, \forall)$, NBD- $C_{6}$ $(-,-)$, and $\mathrm{NBD}_{-} \mathrm{C}_{8}(8,8)$, normalized by the intensity when associated with BSA. The total concentration of NBD- $\mathrm{C}_{4}$ was $100 \mathrm{nM}$, and that of NBD- $\mathrm{C}_{6}$ and $\mathrm{C}_{8}$ was $50 \mathrm{nM}$. Average of at least three replicates of the equilibrium association constant between NBD- $C_{n}$ and BSA, as a function of the length of the alkyl chain, the standard deviation is smaller than the symbol size. The aqueous phase is buffer without (grey symbols) or with $1 \mathrm{M}$ trehalose (black symbols).

The dependence of $K_{\mathrm{B}}$ with the length of the alkyl chain is also shown in Figure 2. A linear dependence of $\ln \left(K_{\mathrm{B}}\right)$ with $n$ was considered to allow prediction of the effect for 
longer alkyl chains. However, small deviations from a linear behavior are already observed pointing towards the expected sigmoid dependence. ${ }^{23}$ Large uncertainties are therefore expected for the values of $K_{\mathrm{B}}$ extrapolated for longer alkyl chains.

Partition of NBD- $C_{n}$ to POPC bilayers. The partition of NBD- $C_{n}$ was first followed via the change in the fluorescence spectra and intensity when associated with the POPC bilayer. ${ }^{23}$ The high sensitivity of this methodology allows the direct characterization for $n=4$ to 10 . When associated with the lipid bilayer, NBD- $\mathrm{C}_{\mathrm{n}}$ fluorescence emission spectra and quantum yield was not significantly affected by the presence of trehalose (results not shown). This is in agreement with the localization of the fluorescent NBD group near the POPC glycerol..$^{30,31}$

The partition coefficient of NBD- $\mathrm{C}_{4}$ and NBD- $\mathrm{C}_{10}$ between the aqueous media and POPC LUVs is shown in Figure 3 , in the temperature range from 15 to $45^{\circ} \mathrm{C}$. Trehalose decreases the partition coefficient of NBD- $\mathrm{C}_{4}$ by 30 to $40 \%$ at all temperatures studied. This behavior agrees with the increased solubility of NBD- $\mathrm{C}_{4}$ in the presence of $1 \mathrm{M}$ trehalose observed at $25^{\circ} \mathrm{C}$, Figure 1 . The effect of trehalose on the partition of NBD- $\mathrm{C}_{10}$ is however unexpected. From the 6-fold increase in its aqueous solubility at $25{ }^{\circ} \mathrm{C}$, one would expect a more significant decrease in the partition coefficient. In contrast, the decrease observed is only $20 \%$ at this temperature. In addition, the decrease observed in the presence of trehalose is dependent on temperature, with no effect being observed at $15{ }^{\circ} \mathrm{C}$. This indicates that the affinity of NBD- $\mathrm{C}_{10}$ for the POPC bilayer is being influenced by additional effects of trehalose, other than through the solubility of the amphiphile in the aqueous phase. This will be addressed below, when characterizing the effect of trehalose on the kinetics of the interaction of NBD- $C_{n}$ with the POPC LUVs.

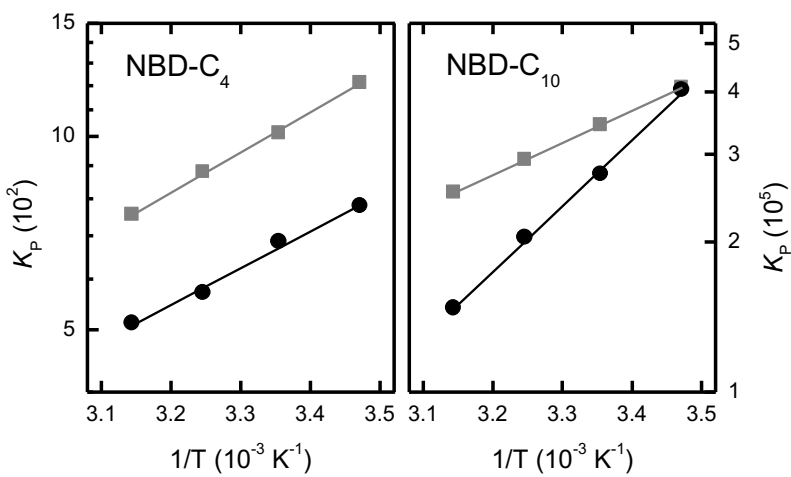

Figure 3 - Temperature dependence of the partition coefficient between the aqueous phase and POPC LUVs, for NBD-C 4 (left) and NBD- $\mathrm{C}_{10}$ (right), in HEPES buffer containing $1 \mathrm{M}$ trehalose (,). The average of 3 to 6 independent experiments is shown. The standard deviation is between 10 and $20 \%$. The results are compared with data from literature in the absence of trehalose $(!)^{23}$

The association of NBD- $C_{n}$ with POPC LUVs was also characterized using Isothermal Titration Calorimetry (ITC). Typical results obtained at $25^{\circ} \mathrm{C}$ are shown in Figure 4. The interaction is exothermic for all amphiphiles studied, $n=4$ to 8 . As observed by fluorescence, the partition coefficient increases with the length of the alkyl chain and is somewhat smaller when the aqueous media contains $1 \mathrm{M}$ trehalose. Due to the low solubility of NBD- $\mathrm{C}_{8}$ in the aqueous media, the concentration cannot go beyond $1 \mu \mathrm{M}$, which leads to a poor signal to noise ratio. Longer alkyl chains cannot be characterized using this technique.

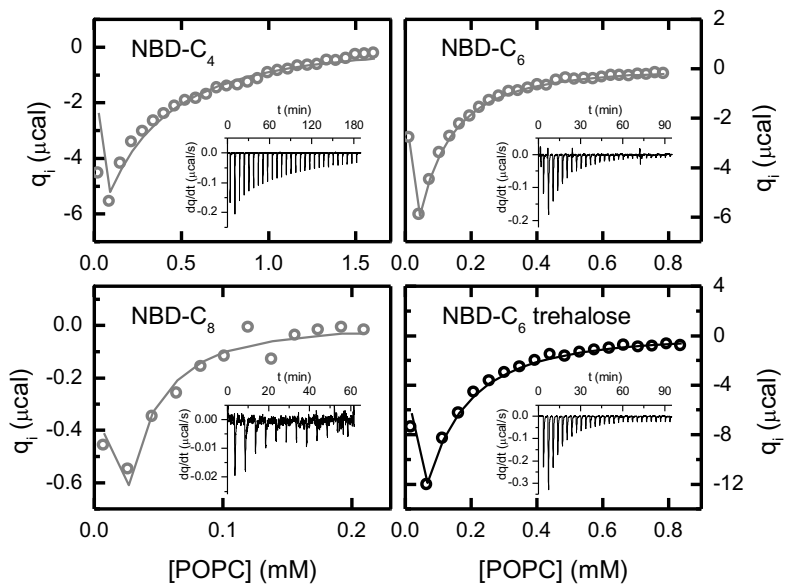

Figure 4 - Heat released due the association of NBD- $C_{n}$ with POPC LUVs at $25{ }^{\circ} \mathrm{C}$. NBD- $C_{n}$ was placed in the cell at a concentration of $30 \mu \mathrm{M}$ (NBD- $\mathrm{C}_{4}$ in HEPES), $10 \mu \mathrm{M}$ (NBD- $\mathrm{C}_{6}$ in HEPES), $15 \mu \mathrm{M}$ (NBD- $\mathrm{C}_{6}$ in $1 \mathrm{M}$ trehalose), and $1 \mu \mathrm{M}$ (NBD- $\mathrm{C}_{8}$ in HEPES), and titrated with POPC LUVs. The insets show the raw thermogram, and the heat per peak in shown in the main plot, together with the best fit according to a simple partition between the aqueous and the lipidic phases with: $K_{\mathrm{P}}=1.7 \times 10^{3}$ and $\Delta H^{\mathrm{o}}=-6.2 \mathrm{~kJ} / \mathrm{mol}$ for $\mathrm{NBD}-\mathrm{C}_{4}$ in HEPES; $K_{\mathrm{P}}=6.7 \times 10^{3}$ and $\Delta H^{\mathrm{o}}=-14 \mathrm{~kJ} / \mathrm{mol}$ for NBD-C $\mathrm{C}_{6}$ in HEPES; $K_{\mathrm{P}}=5.6 \times 10^{3}$ and $\Delta H^{\mathrm{o}}=-16$ $\mathrm{kJ} / \mathrm{mol}$ for $\mathrm{NBD}-\mathrm{C}_{6}$ in trehalose; and $K_{\mathrm{P}}=3.2 \times 10^{4}$ and $\Delta H^{\mathrm{o}}=-$ $10 \mathrm{~kJ} / \mathrm{mol}$ for NBD-C 8 in HEPES.

The partition coefficient obtained at $25{ }^{\circ} \mathrm{C}$ for all amphiphiles using the two methodologies, in the absence and in the presence of trehalose is represented in Figure $5 \mathrm{~A}$. Essentially the same values were obtained by ITC and by changes in the fluorescence intensity, despite the several orders of magnitude difference in the concentrations of amphiphile used in both approaches. A good agreement was also obtained at the other temperatures studied. This indicates that at the local concentrations achieved (less than $0.1 \%$ in fluorescence and less than $4 \%$ in the ITC) the amphiphiles do not perturb significantly the lipid bilayer, as anticipated given their small size and neutral charge. ${ }^{26-}$ 28,31

The results obtained by ITC allow the direct calculation of the enthalpy associated with partition from the aqueous phase into the POPC LUVs $\left(\Delta H^{\circ} \mathrm{cal}\right)$, as well as from the temperature dependence of the partition coefficient $\left(\Delta H^{\circ}\right.$ van't Hoff). The comparison between the two is shown in Figure ${ }_{4} \mathrm{~B}$ for NBD- $\mathrm{C}_{6}$. A monotonic increase with temperature is observed for $\Delta H^{\circ}$ cal (becoming more negative as the temperature increases). The van't Hoff enthalpy variation calculated from the partition coefficient of the same data set is equal to the value of $\Delta H^{\circ}$ cal at the intermediate temperature. This agreement indicates that partition into the POPC bilayer is a simple process, without additional equilibria such as changes in the ionization of the solute upon partition ${ }^{23,32}$. 


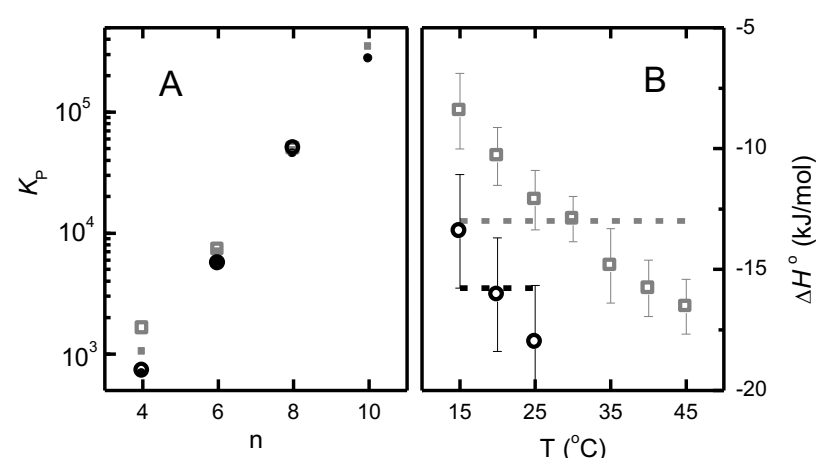

Figure 5 - Plot A: Partition coefficient of NBD-Cn between the aqueous phase and POPC LUVs, as measured by changes in their fluorescence emission $(!,$,$) or by the heat evolved (\forall,-)$. The aqueous phase is buffer without (grey symbols) or with 1 $\mathrm{M}$ trehalose (black symbols). Plot B: Enthalpy variation obtained from the heat evolved due to association of NBD- $\mathrm{C}_{6}$ with POPC LUVs $(\forall,-)$, or from the temperature dependence of the partition coefficient $(, \quad)$.

The more favorable enthalpy variation obtained for NBD- $\mathrm{C}_{6}$ when the aqueous media contains $1 \mathrm{M}$ trehalose, together with the smaller partition coefficient, indicates that the entropy variation upon partition is significantly less favorable. This is in agreement with the increase observed in the solubility of non-polar solutes in aqueous solutions containing trehalose, and is strong evidence for a decrease in the importance of hydrophobic hydration.

The heat capacity change (estimated as $\delta \Delta \mathrm{H} / \delta \mathrm{T}$ ) for the interaction of $\mathrm{NBD}^{-\mathrm{C}_{6}}$ with POPC is $-0.27 \mathrm{~kJ} / \mathrm{mol} / \mathrm{K}$ and $-0.13 \mathrm{~kJ} / \mathrm{mol} / \mathrm{K}$ in the absence and presence of trehalose, respectively. The area of the non-polar region of NBD-C $_{6}$ (the $\mathrm{C}_{6}$ tail) is about $190 \AA^{2}$. Considering a contribution of non-polar surface area to the heat capacity of 1.9 $\mathrm{J} / \mathrm{mol} / \mathrm{K} / \AA^{2}, 33$ the value observed in the absence of trehalose is in very good agreement with the burial of the nonpolar region upon partition to the membrane, this being about half when the aqueous media contains trehalose. This provides additional evidence for the predominant role of the hydrophobic interaction in the interaction of NBD- $\mathrm{C}_{6}$ with POPC membranes in aqueous solution.

Kinetics of the interaction of NBD- $\mathrm{C}_{n}$ with POPC LUVs. The effect of trehalose on the rate of translocation $\left(k_{\mathrm{f}}\right)$ was characterized for NBD- $C_{10}$ and NBD- $C_{16}$. Those alkyl chains were selected due to the complete association with the POPC membrane at relatively small lipid concentrations, ${ }^{23}$ and to be representative of short and long alkyl chain lengths. The slower rate of translocation of NBD- $C_{16}$, allows a more accurate measurement of this rate constant by reaction with dithionite added to the aqueous media outside the LUVs. ${ }^{29}$ For this reason, this system was characterized in detail, while only estimated values have been obtained for NBD- $C_{10}$. The effect of trehalose on the rate constant of translocation is shown in Figure 6 for both amphiphiles, in the temperature range from 15 to $35^{\circ} \mathrm{C}$.

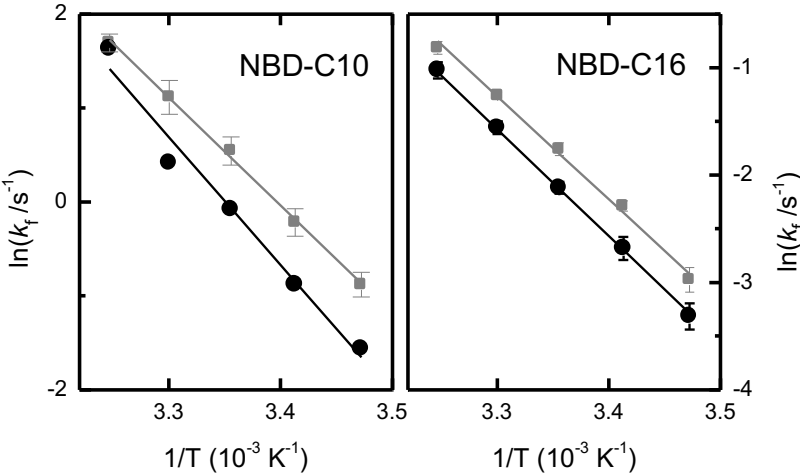

Figure 6 - Average and standard deviation of the results obtained for the rate constant of translocation of NBD- $\mathrm{C}_{10}$ and NBD- $C_{16}$ from the inner to the outer leaflet of POPC LUVs; in aqueous buffer without (! $)^{29}$ or with $1 \mathrm{M}$ trehalose (,). The lines are the best fit of the transition state theory. ${ }^{34}$

A small effect is observed for both amphiphiles, with the rate constant of translocation being smaller in the presence of trehalose. The results are well described by the transition state theory, ${ }^{34}$ with an enthalpy variation associated with the formation of the transition state $\left(\Delta^{\ddagger} H^{\circ}\right)$ equal to $77 \pm 2$ and $82 \pm 2 \mathrm{~kJ} \mathrm{~mol}^{-1}$ in the absence and presence of 1 $\mathrm{M}$ trehalose, respectively, for NBD- $\mathrm{C}_{16}$. A larger value is also obtained for $\Delta^{\ddagger} H^{0}$ in the case of translocation of NBD-C1o in the presence of trehalose. The higher enthalpy variation obtained in the presence of trehalose is the result of a higher effect of trehalose at lower temperatures (about $40 \%$ decrease in $k_{\mathrm{f}}$ at $15{ }^{\circ} \mathrm{C}$ ), which tends to vanish as the temperature increases.

From the best fit of the transition state to the temperature dependence of the rate constant, it should be possible to obtain the entropy variation associated with the formation of the transition state, $\Delta^{\ddagger} S^{0}$. However, this parameter is strongly dependent on the value considered for the transmission coefficient. Although it is not possible to predict the value of this coefficient for a specific transformation, it has recently been shown that for translocation through lipid bilayers it deviates from unity by several orders of magnitude. ${ }^{35}$ Accordingly, we have not proceeded with the calculation of the entropy variation.

The effect of trehalose on the rate of insertion and desorption of NBD- $\mathrm{C}_{10}$ into/from POPC LUVs is shown in Figure 7 . A very significant decrease is observed in the rate of insertion (to about $25 \%$ of its value in the absence of trehalose), and a somewhat smaller decrease in the rate of desorption. The calculated equilibrium association constant $\left(K_{\mathrm{LUV}}=k_{+} / k_{-}\right)$is in fair agreement with the results obtained at equilibrium (Figure 3 ), with a smaller decrease in $K_{\mathrm{Luv}}$ at low temperatures (higher values of $1 / T$ ) due to the almost compensating decrease in $k_{+}$and $k_{\text {- }}$.

The decrease observed in the rate constant of insertion was expected, due to the higher viscosity of the aqueous solution containing trehalose, ${ }^{36,37}$ and to the observation that insertion of NBD- $C_{10}$ into the lipid bilayer of the LUVs is near the diffusion limit $\left(k_{\text {diff }}=2.3 \times 10^{10} \mathrm{M}^{-1} \mathrm{~s}^{-1}\right.$ at $25^{\circ} \mathrm{C}$ considering $\eta=7.5 \mathrm{cP}$ for $1 \mathrm{M}$ trehalose $\left.{ }^{37}\right) .{ }^{29}$ The decrease observed in the rate of desorption is somewhat unexpected. This may reflect small effects on the properties of the POPC bilayer, due to solvation of the po- 
lar lipid head-groups by trehalose. ${ }^{11-15}$ An alternative interpretation is that at the high viscosity of the trehalose solution, the solute is entrapped in the solvent cage around the LUVs for long enough to prevent its diffusion into the bulk solution before re-insertion into the lipid bilayer. The strong temperature effect observed on the rate of desorption (larger decrease in the rate constant at lower temperatures) supports this interpretation because of the high temperature dependence of trehalose viscosity. ${ }^{37}$ The different temperature dependence observed on the rate of insertion and desorption leads to a strong effect of temperature on the variation of the equilibrium partition due to the presence of trehalose (Figure 3).



Figure 7 - Rate constant of insertion (A) and desorption (B) of NBD- $C_{10}$ in POPC LUVs; in aqueous buffer without $(!)^{29}$ or with $1 \mathrm{M}$ trehalose (,). The lines are the best fit of the transition state theory. ${ }^{34}$

Due to the very small aqueous solubility of $\mathrm{NBD}-\mathrm{C}_{14}$, its transfer from the aqueous media into the LUVs cannot be followed directly. Instead, the solute was first equilibrated with BSA, and the transfer from BSA to the lipid bilayer was followed via the increase in the fluorescence intensity of NBD$\mathrm{C}_{14 .}{ }^{29}$ The transfer was characterized at several lipid concentrations, and the best fit of a straight line to the dependence of the characteristic transfer rate constant on the concentration of LUVs allowed the calculation of the rate constant of desorption from the LUVs (intercept $=k-/ 2$ ). The slope is equal to the rate constant of insertion into the LUVs, multiplied by the fraction of NBD- $\mathrm{C}_{14}$ that is free in the aqueous phase. For this, the equilibrium constant for association of NBD- $\mathrm{C}_{14}$ with BSA must be known. The results shown in Figure $8 \mathrm{~A}$, include the rate constant of insertion calculated by assuming that the equilibrium association of $\mathrm{NBD}-\mathrm{C}_{14}$ with BSA is not affected by the presence of trehalose (open circle and dotted line), and considering the prediction extrapolated from the linear fit to the data in Figure 2, $\left(K_{B}^{C_{14}}(\right.$ trehalose $)=0.27 \times K_{B}^{C_{14}}($ HEPES $\left.)\right)$, closed circles and straight line.

A very small decrease is observed in the rate constant of desorption in the presence of trehalose (Figure $8 \mathrm{~B}$ ). When the equilibrium association of NBD- $\mathrm{C}_{14}$ with $\mathrm{BSA}$ is considered unaffected by trehalose, a small decrease is also observed in the rate constant of insertion (Figure 8A, open symbols), leading to an equilibrium association with the LUVs unaffected by the presence of trehalose. However, the results obtained for the equilibrium association of NBD- $C_{4}, C_{6}$ and $C_{8}$ with BSA (Figure 2) predict a smaller value of $K_{\mathrm{B}}$ in the presence of trehalose, mostly due to the higher stability of the amphiphile in the aqueous phase. This smaller $K_{\mathrm{B}}$ leads to a higher fraction of amphiphile free in solution and therefore, in the absence of any other effect, the observed rate of association with the LUVs should have been higher in the presence of trehalose. The rate constant of insertion calculated from the results observed is therefore significantly smaller when this corrected value of $K_{\mathrm{B}}$ is considered.

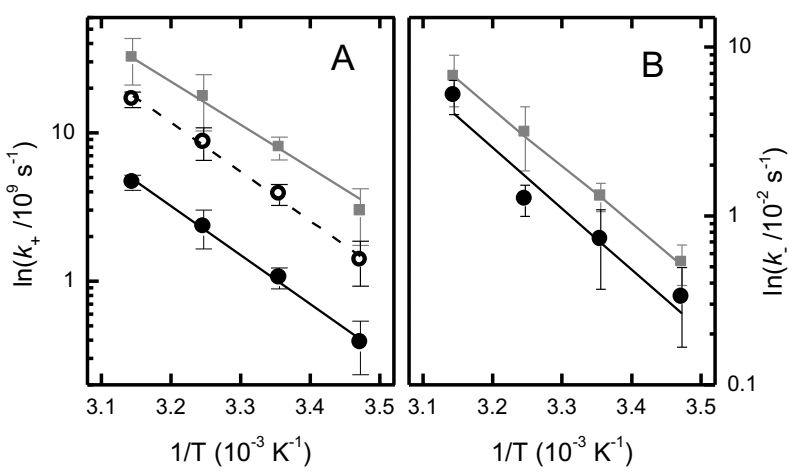

Figure 8 - Rate constant of insertion (A) and desorption (B) of NBD- $\mathrm{C}_{14}$ in POPC LUVs, obtained from the rate of transfer from BSA to the LUVs; in aqueous buffer without $(!)^{29}$ or with $1 \mathrm{M}$ trehalose $(-,$, ,). The lines are the best fit of the transition state theory. ${ }^{34}$ The rate constant of insertion represented by open symbols $(-, \quad)$ ) was calculated from the slope of the characteristic transfer rate constant considering that the equilibrium binding constant to $\mathrm{BSA}\left(K_{\mathrm{B}}\right)$ in trehalose $1 \mathrm{M}$ is equal to that observed in HEPES, while that represented by closed symbols (, - ) was calculated considering that $K_{\mathrm{B}}$ in the presence of $1 \mathrm{M}$ trehalose is equal to that observed in HEPES multiplied by the factor predicted from Figure 2.

The reduction observed on the rate constants of insertion and desorption is similar at all temperatures. Therefore, it is predicted that the presence of trehalose leads to no effect or a small decrease in the equilibrium association of NBD$\mathrm{C}_{14}$ with the POPC LUVs $\left(K_{\mathrm{LUV}}=k_{+} / k_{-}\right)$at all temperatures. A similar effect at all temperatures was also observed for NBD- $C_{4}$, but not for NBD- $C_{10}$, for which the decrease was more accentuated at higher temperatures. The molecular interpretation of this observation is not yet completely understood. The observation that the rate of interaction of NBD- $\mathrm{C}_{10}$ with the POPC bilayers is near diffusion control and closer to this limit at higher temperatures may provide some explanation. In this context it is predicted that interactions that occur at rates close to or at the diffusion limit will be strongly decreased by the presence of trehalose, both on the rate at which they occur and on the equilibrium extent.

\section{Concluding remarks}

This work presents an extensive characterization of the effects of the presence of trehalose on the solubility of a homologous series of amphiphiles in aqueous media and their association with a soluble protein (BSA) and lipid membranes (POPC LUVs).

It is observed that at $1 \mathrm{M}$, trehalose significantly decreases the magnitude of the hydrophobic effect, with a corresponding increase in the solubility of non-polar molecules. The increased stability of the non-polar molecules in the aqueous media leads to a decrease in the equilibrium association with hydrophobic assemblies, such as proteins and 
lipid bilayers. The decrease in the magnitude of the hydrophobic effect is also observed in the enthalpy and entropy contribution to the association of the amphiphiles with lipid bilayers, with a more favorable enthalpy contribution in the presence of trehalose, and a more favorable entropy contribution in its absence.

The presence of high trehalose concentrations in the aqueous media leads to a significant increase in the viscosity of the solutions which influences the kinetics (and indirectly the equilibrium) of the association of the amphiphiles with binding agents. When the rate constants of association/dissociation (insertion/desorption) are much smaller than that expected for a diffusion-controlled process, no significant effects are observed. However, a substantial slowdown of the kinetics is observed for processes close to being diffusion limited. The results obtained indicate a different magnitude for the effect on the rate of association (a second order process), than on the rate of dissociation (a first order process), thus resulting in non-proportional variation on the equilibrium association.

Translocation between the lipid bilayer leaflets is not expected to be influenced by the properties of the aqueous media. However, a small decrease in the rate of this process is observed. This may result from interactions established between the amphiphile and the aqueous media, when at the equilibrium position in the bilayer, or from changes in the properties of the lipid bilayer. Molecular dynamics simulations of NBD- $C_{n}$ inserted in POPC bilayers reveals the establishment of hydrogen bonds between water and the oxygens in the polar portion of the amphiphiles. ${ }^{31}$ The presence of trehalose in the aqueous media may influence the number and strength of these interactions with effects on the kinetics of the translocation process.

This work predicts that the distribution of amphiphiles in high molecular crowded biological media will be significantly different from that predicted in dilute aqueous solutions, with effects on both the kinetics and equilibrium of the interactions established.

\section{Acknowledgment}

This work was partially supported by the Portuguese "Fundação para a Ciência e a Tecnologia" (FCT) through projects $007630 \quad$ UID/QUI/oo313/2013, PT2020_PTDC_DTP-FTO_2784_2014, co-funded by COMPETE2020-UE, and CENTRO-01-0145-FEDER-0ooo14 through "Programa Operacional Regional do Centro" CENTRO2O20.

\section{References}

(1)Gekko, K.; Timasheff, S. N. Biochemistry 1981, 20, 4667.

(2)Lee, J. C.; Timasheff, S. N. J. Biol. Chem. 1981, 256, 7193.

(3)Arakawa, T.; Timasheff, S. N. Biochemistry 1982, 21, 6536.

(4)Arakawa, T.; Timasheff, S. N. Biophys. J. 1985, 47, 411.

(5)Timasheff, S. N. Proc. Natl. Acad. Sci. USA 2002, 99, 9721.

(6)Timasheff, S. N. Biochemistry 2002, 41, 13473.

(7)Arakawa, T.; Timasheff, S. N. Archives of Biochemistry and Biophysics 1983, 224, 169.
(8)Kiselev, M. A.; Zbytovska, J.; Matveev, D.; Wartewig, S.; Gapienko, I. V.; Perez, J.; Lesieur, P.; Hoell, A.; Neubert, R. Colloids Surf., A 2005, 256, 1.

(9)Koynova, R.; Brankov, J.; Tenchov, B. Eur. Biophys. J. Biophys. Lett. 1997, 25, 261.

(10) Crowe, L. M.; Crowe, J. H. Biochim. Biophys. Acta 1991, 1064, 267.

(11) Diaz, S.; Lairion, F.; Arroyo, J.; de Lopez, A. C. B.; Disalvo, E. A. Langmuir 2001, 17, 852.

(12) Leekumjorn, S.; Sum, A. K. Mol. Simul. 2006, 32, 219.

(13) Sum, A. K.; Faller, R.; de Pablo, J. J. Biophys. J. 2003, 85, 2830

(14) Villarreal, M. A.; Diaz, S. B.; Disalvo, E. A.; Montich, G. G. Langmuir 2004, 20, 7844.

(15) Skibinsky, A.; Venable, R. M.; Pastor, R. W. Biophys. J. 2005, $89,4111$.

(16) Ricker, J. V.; Tsvetkova, N. M.; Wolkers, W. F.; Leidy, C.; Tablin, F.; Longo, M.; Crowe, J. H. Biophys. J. 2003, 84, 3045.

(17) Cacela, C.; Hincha, D. K. Biophys. J. 2006, 90, 2831.

(18) Ferreira, L. A.; Breydo, L.; Reichardt, C.; Uversky, V. N.; Zaslavsky, B. Y. Journal of Biomolecular Structure \& Dynamics 2017, 35, 1055.

(19) Bai, G.; Nunes, S. C. C.; Rocha, M. A. A.; Santos, L. M. N. B. F.; Eusebio, M. E. S.; Moreno, M. J.; Bastos, M. Pure And Applied Chemistry 2014, 86, 223.

(20) Francois, J.; Parrou, J. L. Fems Microbiology Reviews 2001, 25, 125.

(21) Nunes, S. C. C.; Jesus, A. J. L.; Moreno, M. J.; Eusebio, M. E. S. Carbohydrate Research 2010, 345, 2048.

(22) Winther, L. R.; Qvist, J.; Halle, B. J. Phys. Chem. B 2012, $116,9196$.

(23) Cardoso, R. M. S.; Filipe, H. A. L.; Gomes, F.; Moreira, N. D.; Vaz, W. L. C.; Moreno, M. J. J. Phys. Chem. B 2010, 114, 16337.

(24) Peters, T. All about Albumin. Biochemistry, Genetics, and Medical Applications; Academic Press: New York, 1996.

(25) Moreno, M. J.; Estronca, L. M. B. B.; Vaz, W. L. C. Biophysical Journal 2006, 91, 873.

(26) Samelo, J.; Mora, M. J.; Granero, G. E.; Moreno, M. J. ACS Omega 2017, 2, 6863.

(27) Moreno, M. J.; Bastos, M.; Velazquez-Campoy, A. Anal. Biochem. 2010, 399, 44.

(28) Martins, P. T.; Velazquez-Campoy, A.; Vaz, W. L. C.; Cardoso, R. M. S.; Valerio, J.; Moreno, M. J. J. Am. Chem. Soc. 2012, 134, 4184.

(29) Cardoso, R. M. S.; Martins, P. A. T.; Gomes, F.; Doktorovova, S.; Vaz, W. L. C.; Moreno, M. J. J. Phys. Chem. B 2011, 115, 10098.

(30) Amaro, M.; Filipe, H. A. L.; Ramalho, J. P. P.; Hof, M.; Loura, L. M. S. Phys. Chem. Chem. Phys. 2016, 18, 7042.

(31) Filipe, H. A. L.; Moreno, M. J.; Loura, L. M. S. J. Phys. Chem. B 2011, 115, 10109.

(32) Coreta-Gomes, F. M.; Martins, P. A. T.; VelazquezCampoy, A.; Vaz, W. L. C.; Geraldes, C. F. G.; Moreno, M. J. Langmuir 2015, 31, 9097.

(33) Murphy, K. P.; Freire, E. In Advances in Protein Chemistry; Anfinsen, C. B., Richards, F. M., Edsall, J. T., Eisenberg, D. S., Eds.; Academic Press: 1992; Vol. 43, p 313.

(34) Steinfeld, J. I.; Francisco, J. S.; Hase, W. L. Chemical Kinetics and Dynamics; 2nd ed.; Prentice-Hall: New Jersey, 1999.

(35) Filipe, H. A. L.; Javanainen, M.; Salvador, A.; Galvão, A. M.; Vattulainen, I.; Loura, L. M. S.; Moreno, M. J. Journal of Chemical Theory and Computation 2018.

(36) Longinotti, M. P.; Corti, H. R. J. Phys. Chem. Ref. Data 2008, 37, 1503.

(37) Magazù, S.; Maisano, G.; Migliardo, P.; Middendorf, H. D.; Villari, V. The Journal of Chemical Physics 1998, 109, 1170. 
